\title{
A reemergência do sarampo no Brasil associada à influência dos movimentos sociais de pós verdade, fake news e antivacinas no mundo: revisão integrativa
}

\author{
The reemergence of sarampo in Brazil associated with the influence of social movements \\ of post truth, fake news and antivacines in the world: integrative review
}

La reemergencia del sarampo en Brasil asociada a la influencia de los movimientos sociales de post verdad, falsas noticias y antivacinas en el mundo: revisión integrativa

Hatus da Silva Almeida ${ }^{1}$, Sueli de Souza Costa ${ }^{1}$, Izolda Souza Costa ${ }^{2 \star}$, Claudio Rogério Rocha Junior ${ }^{3}$.

\section{RESUMO}

Objetivo: Analisar os trabalhos publicados envolvendo os recentes surtos de sarampo e sua relação com o fenômeno recente de pós-verdade, fake news e movimento antivacinas. Métodos: Revisão de literatura integrativa, nas bases de dados eletrônicas LILACS, PUBMED e SCIELO, com os seguintes descritores, em língua portuguesa: "sarampo"; "vacinação"; "notícias"; "recusa de vacinação" e "detecção de mentiras", no período de 2013 a 2019. Foram empregadas etapas de combinações entre os descritores, em categorias, para a seleção dos artigos e análise. Resultados: Foram encontrados 3680 artigos, sendo 12 da base de dados SCIELO, 3639 no PUBMED e 29 no LILACS, que, após a aplicação dos critérios de exclusão, restaram 60 artigos. O Brasil aparece em três publicações (5\% do total). Observou-se um crescimento linear entre as publicações, dos anos de 2013 a 2019. Considerações finais: A produção acadêmica mundial quanto ao tema, foi significativa no período, quando observado os componentes da ideia proposta de forma isolada, apontando alguns assuntos com maior ênfase que outros. Observa-se, entretanto, a necessidade de mais publicações envolvendo as temáticas de forma integrada, afim de que se possa estabelecer, ou não, associação dos termos na dinâmica causa-consequência.

Palavras-chave: Sarampo, Saúde, Sistema Único de Saúde, Movimento contra vacinação, Saúde pública.

\begin{abstract}
Objective: To analyze published works involving the recent measles outbreaks and their relationship with the recent phenomenon of post-truth, fake news and anti-vaccine movement. Methods: Integrative literature review, in the electronic databases LILACS, PUBMED and SCIELO, with the following descriptors, in Portuguese: "measles"; "vaccination"; "News"; "Refusal of vaccination" and "detection of lies", from 2013 to 2019. Combination steps between descriptors, in categories, were used for the selection of articles and analysis. Results: 3680 articles were found, 12 from the SCIELO database, 3639 from PUBMED and 29 from LILACS, which, after applying the exclusion criteria, left 60 articles. Brazil appears in three publications (5\% of the total). There was a linear growth between publications, from the years 2013 to 2019 . Final considerations: The worldwide academic production regarding the theme, was significant in the period, when the components of the proposed idea were observed in isolation, pointing out some subjects with greater emphasis what others. However, there is a need for more publications involving the themes in an integrated manner, in order to establish, or not, an association of terms in the cause-consequence dynamic.
\end{abstract}

Key words: Measles, Cheers, Health Unic System, Movement against vaccination, Publich health.

\section{RESUMEM}

Objetivo: Analizar los trabajos publicados sobre los recientes brotes de sarampión y su relación con el fenómeno reciente de postverdad, fake news y movimiento antivacunas. Métodos: Revisión integrativa de la literatura, en las bases de datos electrónicas LILACS, PUBMED y SCIELO, con los siguientes descriptores, en portugués: "sarampión"; "vacunación"; "Noticias"; "Rechazo de vacunación" y "detección de mentiras", de 2013 a 2019. Se utilizaron pasos de combinación entre descriptores, en categorías, para la selección de artículos y análisis. Resultados: Se encontraron 3680 artículos, 12 de la base de datos SCIELO, 3639 de PUBMED y 29 de LILACS, que tras aplicar los criterios de exclusión dejaron 60 artículos. Brasil aparece en tres publicaciones ( $5 \%$ del total). Hubo un crecimiento lineal entre las publicaciones, de los años 2013 a 2019. Consideraciones finales: La producción académica mundial en torno al tema, fue significativa en el período, al observar los componentes de la idea propuesta de forma aislada, señalando algunos temas con mayor énfasis Que otros. Sin embargo, se necesitan más publicaciones que involucren los temas de manera integrada, a fin de establecer, o no, una asociación de términos en la dinámica causa-consecuencia.

Palabras clave: Sarampión, Salud, Sistema Único de Salud, Movimiento contra la vacunación, Salud pública.

\footnotetext{
1 Universidade Federal do Maranhão (UFMA), Pinheiro - MA.

2 Universidade Federal do Maranhão (UFMA), São Luís - MA. *E-mail: izolda.costa@hotmail.com

3 Universidade Positivo (UP), Curitiba - PR.
} 


\section{INTRODUÇÃO}

Sarampo é doença infecciosa aguda, contagiosa, transmitida através do contato direto com secreções nasofaringeas advindas de pacientes infectados, e cuja prevenção é através da vacinação (BRANCO VGC e MORGADO FEF, 2019; XAVIER AR, et al., 2019).

A vacina Tríplice Viral, que protege contra sarampo, rubéola e caxumba, é disponibilizada pelo Sistema Único de Saúde (SUS), sendo que o Ministério da Saúde recomenda que todos indivíduos de 1 a 29 anos de idade recebam duas doses da vacina. Vários surtos ocorreram, acompanhados de campanhas intensas de vacinação e combate à doença, sendo que no ano 2000 registrou-se os últimos casos nativos da doença, reaparecendo um surto em 2013, que se estendeu até 2015, associada a movimentos relacionados a mídias sociais de relacionamento e envio de mensagens: fake news, pós-verdade e antivacinação (NAZARETH RT, 2019; HENRIQUES CMP, 2018).

Esses movimentos ganharam notoriedade em 2016, devido ao número de informações falsas em mídias sociais, sendo a área das vacinas um alvo constante, tornando-se problema de saúde pública, uma vez que o uso de redes sociais para divulgação de informações não verídicas e que conferem perigo aos usuários, permite disseminação em larga escala (MELLO JN, et al., 2014).

Como resposta às fake news sobre a saúde, o Ministério da Saúde criou em 2018 um canal de comunicação por meio do aplicativo WhatsApp®, para que a população, ao se deparar com informações de caráter duvidoso, possa envia-las para ao canal criado avalia-las quanto à veracidade. O resultado da avaliação é repassado aos usuários e disponibilizado no site do Ministério. Dessa maneira, seria possível compartilhar a informação de forma segura (DA SILVA FV e DA SILVA JÚNIOR J, 2019; GONZAGA JD e DOS SANTOS AOC, 2019).

Sarampo é doença que ainda permanece como problema de saúde pública, principalmente em áreas de ocorrência endêmica, como Ásia e Europa. Entre 2000 e 2017, a incidência anual global de casos havia diminuído em $83 \%$ (de 145 para 25 casos por milhão de habitantes), reduzindo-se também a mortalidade neste mesmo período: 109.000 mortes em 2017, sendo 545.000 em 2000 (SILVA SS, 2018).

Entretanto ocorreu aumento considerável no número de casos em 2018 e 2019 (GONZANGA, 2019). Entre os países com maior número de notificações nesse período estão Índia, Ucrânia, Madagascar, Filipinas, Tailândia, Cazaquistão, Nigéria, Paquistão, Venezuela e Brasil. Esses casos notificados, por mais que tenham relação com sistemas de saúde deficientes, estão em sua maioria relacionados à hesitação e recusa da vacina, sendo esse fenômeno apontado pela Organização Mundial de Saúde (OMS) como uma das 10 principais ameaças à saúde em 2019 (BRANCO VGC e MORGADO FEF, 2019; GONZAGA JD e DOS SANTOS AOC, 2019).

Observando o Brasil, verifica-se a ocorrência de surtos em 2013 no estado de Pernambuco e em 2014 no Ceará, com a aplicação de medidas de controle efetiva, o que fez com o país recebesse da Organização PanAmericana da Saúde (OPAS), em 2016, o certificado de eliminação da circulação do vírus, concedendo o título de região das Américas livre da doença (SILVA SS, 2018). Entretanto, em 2018 novos surtos sugiram, nos estados do Amazonas, Roraima, Pará, São Paulo, e a doença tem se espalhado de forma rápida. Observou-se também nesse período (2013-2018) uma queda na cobertura vacinal para o sarampo, mais significativa na região norte do país (SILVA SS, 2018).

No Brasil, os surtos de 2013 em diante podem ser relacionados a inúmeras possibilidades. Uma delas, apontadas pelo Ministério da Saúde em 2018 através de nota, é a veiculação de fake news através de redes sociais e aplicativos de troca de mensagens como WhatsApp, Twitter e Facebook, responsáveis pela queda de $70 \%$ a 75\% na cobertura vacinal desde 2016 (BRANCO VGC e MORGADO FEF, 2019).

A vacinação é a forma de prevenção mais eficaz e a primeira vacina contra o sarampo foi desenvolvida em 1963. Na década de 70, a OMS recomendou a vacinação sistemática com dose única nas crianças com mais de 9 meses de idade (BRASIL, 2018a). No Brasil, a vacinação tornou-se obrigatória e regulada pelo Decreto 78.231, de 12 de agosto de 1976 (BRANCO VGC e MORGADO FEF, 2019; BRASIL, 2019). 
A portaria de 17 de fevereiro de 2016, do Ministério da Saúde brasileiro, tornou o sarampo doença de notificação obrigatória, incluindo-o na Lista Nacional de Notificação Compulsória de Doenças (SBIM, 2018; BRASIL, 2019).

A maior epidemia registrada do sarampo no Brasil, ocorreu em 1986, com 129.942 casos, representando uma taxa de incidência de 97,7/100.000 hab, sendo que na década de 80 foram registrados 15.638 óbitos. Nos anos seguintes foram realizadas campanhas de vacinação em massa contra o sarampo. Em 1997, ocorreu uma nova epidemia em vários estados brasileiros, com cerca de 91.810 casos notificados e 53.664 confirmados, sendo a taxa de incidência de 32,6/100.000 habitantes e 61 óbitos. Com a implantação do Plano de Eliminação do Sarampo, em 2000, os números relacionados à doença sofreram queda expressiva. A partir de 2001, ocorreram casos importados, mas sem grande magnitude e controlados pelas ações de prevenção e controle (XAVIER AR, et al., 2019).

Entre 2013 e 2015, novos surtos ocorreram, em Pernambuco e no Ceará. Em 2016, o Brasil recebeu o certificado de eliminação da circulação do vírus do sarampo pela OMS. Entretanto, em janeiro de 2018, casos do tipo importados, advindos da Venezuela, promoveram novo surto, nos estados de Roraima e no Amazonas. Alguns outros casos confirmados ocorreram no Rio Grande do Sul, São Paulo, Mato Grosso e Rio de Janeiro (DA SILVA FV e DA SILVA JÚNIOR J, 2019; BRANCO VGC e MORGADO FEF, 2019; SBIM, 2018).

O surto atual do sarampo no país pode ser relacionado à baixa adesão da vacina, por motivos como crenças pessoais, religiosas e notícias falsas divulgadas e movimentos sociais como antivacinação; baixa cobertura vacinal, principalmente em países em desenvolvimento; dificuldade no controle global da doença; implicações dos novos surtos de sarampo na prática clínica e a necessidade de adaptação dos profissionais de saúde envolvidos no controle (RIBEIRO C, et al., 2015).

Os termos pós-verdade e fake news estão em voga desde 2016 devido a veiculação de um número exorbitante de informações falsas em sites e diferentes mídias sociais, que levaram a opinião pública a tomar decisões equivocadas, baseadas na emoção e em crenças pessoais, ao invés de buscar fundamentação em fatos objetivos (GENESINI S, 2018; SILVA LM, et al., 2017). A procura por informações em saúde atualmente tem como fonte principal a Internet e, apesar desta proporcionar a democratização da informação, não ocorre o mesmo com a motivação e formação dos usuários na validação dos assuntos pesquisados (MANSO MEG, et al., 2019; NAZARETH RT, 2019; GONZAGA JD e DOS SANTOS AOC, 2019; SILVA LM, et al., 2017).

As fake news também são veiculadas por ferramentas chamadas "bots" (robôs), contas automatizadas que por uso de algoritmos relacionados aos padrões de uso dos usuários, podem influenciar o comportamento nas redes (MANSO MEG, et al., 2019).

A exploração de temas polêmicos na área de saúde pode ocasionar danos severos. Quanto à vacinação, observada na campanha de imunização da febre amarela em 2018, em meio a um surto da doença, parte da população hesitou em se vacinar por acreditar que a mesma poderia ser mais letal do que uma infecção pela própria doença (HENRIQUES CMP, 2018).

A publicação de um artigo no periódico inglês The Lancet, em 1988, associava casos de autismo e doença inflamatória intestinal com uso da vacina tríplice viral (sarampo, caxumba e rubéola) (WAKEFIELD AJ, et al., 1998). A notícia promoveu enorme repercussão no meio científico e no público leigo, resultando em contraindicações dos médicos e recusa dos pais em aplicar em seus filhos a vacina, promovendo um número significativo de surtos dessas viroses. Em 2010, o equívoco na notícia foi apresentado pelo próprio veículo de informação. Entretanto, as consequências eram muitas, dentre elas, a formação de grupos contrários à vacinação (LAGO EG, 2018; SILVA LM, et al., 2017).

Dentro desse fenômeno, é possível observar duas linhas de pensamento: a hesitação vacinal, caracterizada por pessoas que atrasam a vacinação ou recusam alguns tipos de vacinas, e a recusa vacinal, que se trata de indivíduos que rejeitam todo e qualquer tipo de vacina. Os motivos são diversos: filosóficos ou religiosos, aspectos socioculturais, falta de informação sobre o risco de doenças, indagações sobre orientação médica (SATO APS, 2018). 
No entanto, esse fenômeno não é recente. No Brasil, o episódio da Revolta da Vacina, ocorrido no Rio de Janeiro em 1904, com a participação de Oswaldo Cruz, promoveu medidas higienistas como a vacinação obrigatória contra varíola, resultando na insatisfação e posterior revolta da população (SAITA HS, 2019). Com o advento da internet e das redes sociais, esse fenômeno aumentou exponencialmente. O Ministério da Saúde do Brasil, em setembro de 2018, divulgou nota relatando que uso de redes sociais e aplicativos de troca de mensagens como WhatsApp $\Theta$, Twitter $\Theta$ e Facebook $\Theta$, por meio de veiculação de notícias falsas, exerceram influência na cobertura das campanhas de vacinação promovidas desde 2016, apontando o fenômeno como responsável pela queda de $70 \%$ a $75 \%$ no alcance das ações de imunização (BRASIL, 2018b; DA SILVA FV e DA SILVA JÚNIOR J, 2019).

Em resposta às ondas de fake news e pós-verdade, o Ministério da Saúde, em 2018, lançou o programa "Saúde sem Fake News", com o objetivo de checagem por profissionais de informações divulgadas nos veículos de relacionamento e troca de mensagens. Após a checagem pelos responsáveis técnicos sobre se há comprovação científica acerca das mensagens, as mesmas são colocadas no Portal da Saúde, juntamente com o selo "Isto é fake news!" ou "Esta notícia é verdadeira", a depender do resultado da verificação (DA SILVA FV e DA SILVA JÚNIOR J, 2019; GONZAGA JD e DOS SANTOS AOC, 2018).

Assim, em vista da escassez de artigos na área, o objetivo desta pesquisa é realizar revisão de literatura integrativa sobre os trabalhos publicados, no Brasil e no mundo, envolvendo os recentes surtos de sarampo e sua relação com o fenômeno recente de pós-verdade, salientando a questão de fake news e movimento antivacinas, no período de 2013 a 2019.

\section{MÉTODOS}

Trata-se de revisão de literatura integrativa, realizada em bases de dados eletrônicas LILACS, PUBMED, SCIELO, no período de 2013 a 2019, utilizando-se o diagrama de fluxo de revisão PRISMA 2009 (MOTHER $\mathrm{D}$, et al., 2009). As referências que preencheram os critérios de inclusão foram avaliadas com relação ao conteúdo publicado e estrutura do trabalho. A seleção dos descritores utilizados no processo de revisão foi efetuada mediante consulta ao DECs (Descritores de Assunto em Ciências da Saúde, da BIREME). Foram incluídos, para a busca, os seguintes descritores, em língua portuguesa e inglesa: "sarampo"; "vacinação"; "notícias", "recusa de vacinação" e "detecção de mentiras".

Foram utilizados etapas e descritores em categorias para a seleção dos artigos, nas bases de dados, em três combinações, com quatro categorias de descritores em arranjo, sendo: 1- Uso dos descritores: "sarampo", "vacinação" e "noticias", dispostos da seguinte forma: "sarampo" + "vacinação"; "sarampo" + "notícias"; e "vacinação" + "notícias"; e "sarampo" + "vacinação" + "notícia"; 2- Uso dos descritores: "sarampo", "recusa de vacinação" e "noticias", dispostos da seguinte forma: "sarampo" + "recusa de vacinação"; e "recusa de vacinação" + "notícias"; e "sarampo" + "recusa de vacinação" + "notícia"; 3- Uso dos descritores: "sarampo", "recusa de vacinação" e "detecção de mentiras", dispostos da seguinte forma: "sarampo" + "detecção de mentiras"; e "recusa de vacinação" +"detecção de mentiras"; e "sarampo" + "recusa de vacinação" + "detecção de mentiras".

Foram incluídos artigos científicos, na íntegra (open acess), que abrangessem os termos determinados pelos descritores; publicações originais de pesquisa, artigo científicos, teses, dissertações e monografias; no período de 2013 até 2019; português, inglês e espanhol; onde o descritor "notícias" estaria relacionado aos termos fake news e pós-verdade. Foram excluídos artigos que, após busca por combinação dos descritores, atenderam a somente um dos descritores usados, ou que fugissem ao tema enfocado.

$\mathrm{Na}$ avaliação dos artigos, foram observados os seguintes aspectos: amostra (ano de publicação, autores, local de realização do trabalho); metodologia (informações sobre a forma de avaliação das amostras utilizadas nos trabalhos, independentemente do tipo); conclusão dos trabalhos selecionados. Foi realizada a tabulação dos trabalhos encontrados e análise para descrição nos resultados.

\section{RESULTADOS}

No início da pesquisa, com o uso dos descritores selecionados, foram encontrados 3680 artigos, sendo 12 da base de dados SCIELO, 3639 no PUBMED e 29 no LILACS (Tabela 1). 
Tabela 1 - Disposição do número de artigos conforme arranjos dos descritores usados na pesquisa em base de dados.

\begin{tabular}{cccc}
\hline Combinação & SCIELO & PUBMED & LILACS \\
\hline Sarampo e vacinação & 12 & 2698 & 27 \\
Sarampo e notícias & 0 & 188 & 0 \\
Vacinação e notícias & 0 & 488 & 0 \\
Sarampo e vacinação e notícias & 0 & 49 & 0 \\
Sarampo e recusa de vacinação & 0 & 192 & 2 \\
Recusa de vacinação e notícias & 0 & 18 & 0 \\
Sarampo e recusa de vacinação e notícias & 0 & 6 & 0 \\
Sarampo e detecção de mentiras & 0 & 0 & 0 \\
Recusa de vacinação e detecção de mentiras & 0 & 0 & 0 \\
Sarampo e recusa de vacinação e detecção de mentiras & 0 & 0 & 0 \\
\hline Total & $\mathbf{1 2}$ & $\mathbf{3 6 3 9}$ & $\mathbf{2 9}$ \\
\hline
\end{tabular}

Fonte: Almeida HS, et al., 2021.

Após a leitura analítica dos artigos, aplicando os critérios de inclusão e exclusão, resultaram na amostra final 60 artigos. Foram encontrados trabalhos nas línguas portuguesa $(n=2)$ e língua inglesa $(n=58)$. $O$ ano de 2019 foi o de maior número de publicações (31,66\%), seguido de 2018 (21,66\%) (Gráfico 1).

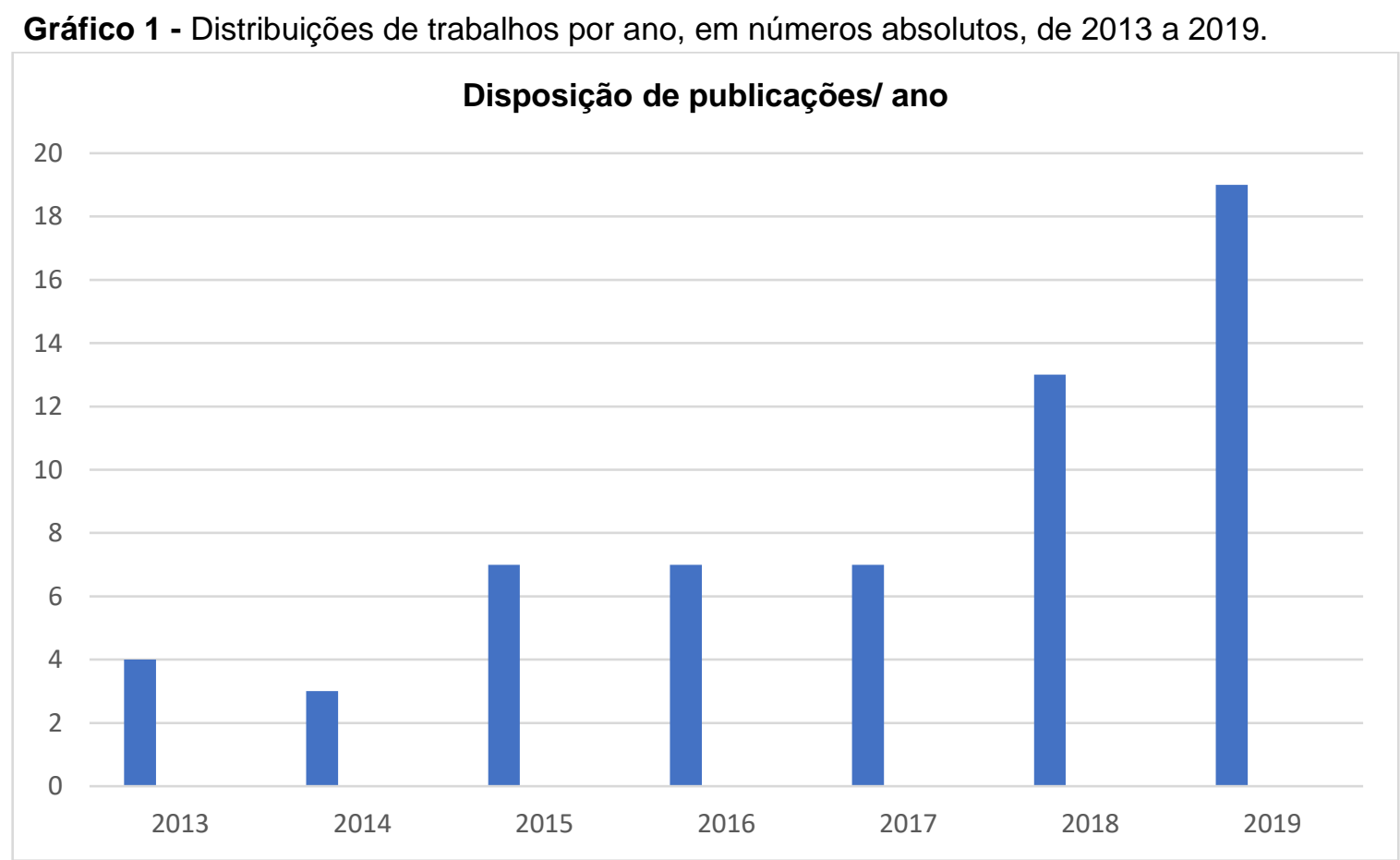

Fonte: Almeida HS, et al., 2021.

Os locais com maiores números de publicações foram os Estados Unidos $(31,66 \%)$, seguido da Itália $(18,33 \%)$. O Brasil teve três publicações, representando $5 \%$, resultado semelhante ao Canadá (Tabela 2). Dentre as publicações nacionais, $66,66 \%$ eram do Rio de Janeiro, e 34\% de São Paulo. Quando se analisa conjuntamente anos de publicação e países, observa-se que 2018 e 2019, além de possuírem o maior número de publicações, também apresentam a maior variedade de países de origem, sendo 2018 com 11 localidades diversas e 2019 com nove (Gráfico 2). 
Tabela 2 - Número de publicações de trabalhos por países em números absolutos e percentuais.

\begin{tabular}{ccc}
\hline País & Número de publicações em absoluto & $\begin{array}{c}\text { Número de publicações em } \\
\text { percentual }\end{array}$ \\
\hline Austrália & 1 & $1,66 \%$ \\
Áustria & 1 & $1,66 \%$ \\
Brasil & 3 & $5 \%$ \\
Canadá & 3 & $5 \%$ \\
Cracóvia & 1 & $1,66 \%$ \\
Dinamarca & 1 & $1,66 \%$ \\
Escócia & 1 & $1,66 \%$ \\
EUA & 19 & $31,66 \%$ \\
França & 2 & $3,33 \%$ \\
Índia & 4 & $6,66 \%$ \\
Israel & 3 & $5 \%$ \\
Itália & 11 & $18,33 \%$ \\
Omã & 1 & $1,66 \%$ \\
Países baixos & 1 & $1,66 \%$ \\
Portugal & 1 & $1,66 \%$ \\
Reino unido & 2 & $3,33 \%$ \\
Romênia & 1 & $1,66 \%$ \\
Sudão & 1 & $1,66 \%$ \\
Suécia & 1 & $1,66 \%$ \\
Suíça & 2 & $3,33 \%$ \\
\hline Total & 60 & $\mathbf{1 0 0}$ \\
\hline Almeida & &
\end{tabular}

Fonte: Almeida HS, et al., 2021.

Gráfico 2 - Distribuições de trabalhos por ano, em números absolutos, de acordo com o país de origem, de 2013 a 2019.

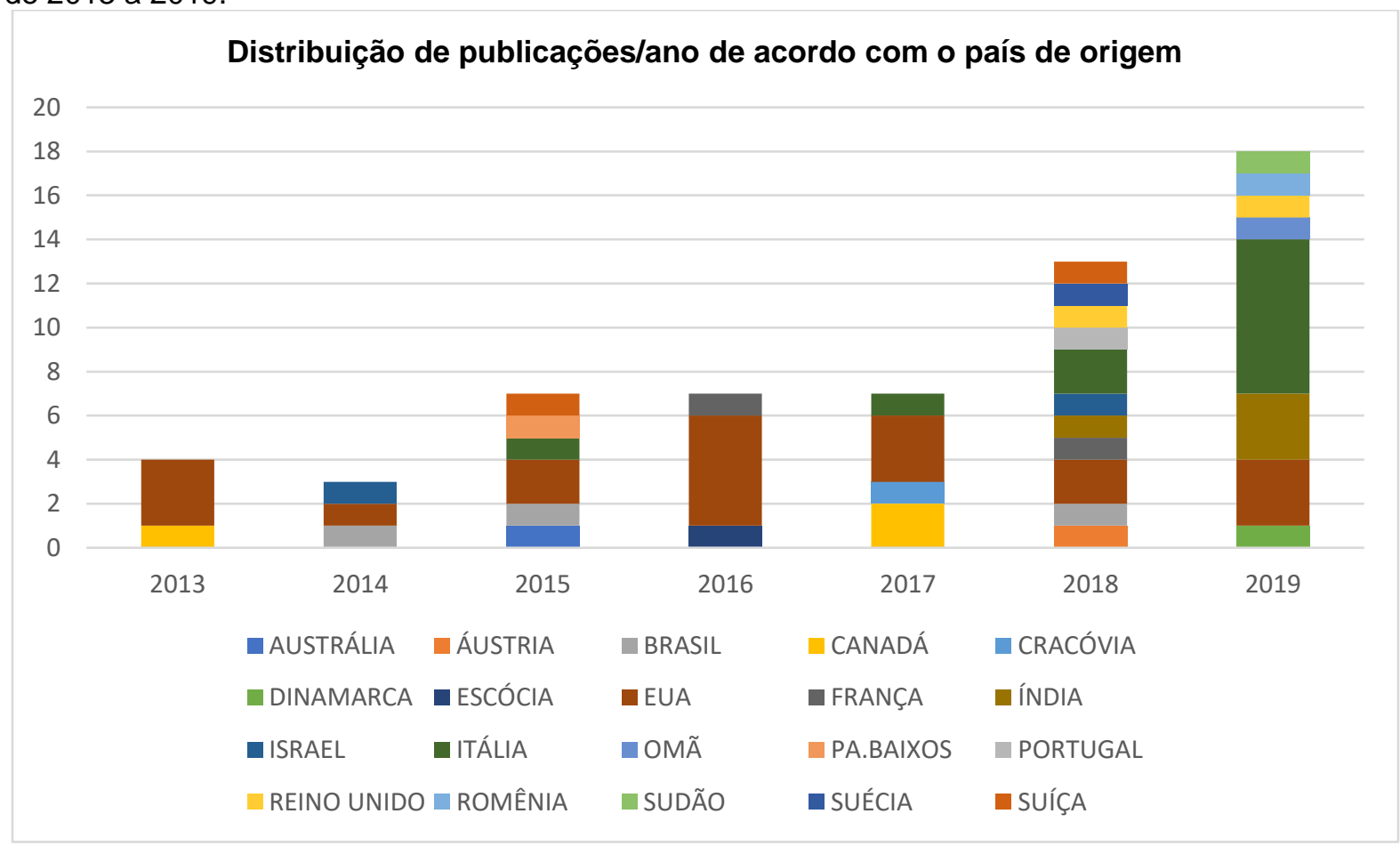

Fonte: Almeida HS, et al., 2021. 
Com relação aos tipos de publicações, os trabalhos de pesquisa aplicados (exploratória, quantitativadescritiva e experimental) representaram a maior percentagem (75\%), sendo os demais do tipo revisão de literatura, variando em sua tipologia (narrativa, sistemática e integrativa). Os demais dados, assim como a síntese do processo de trabalho dos dados coletados, estão apresentados no item Discussão e no fluxograma da Figura 2.

Figura 2 - Fluxograma do processo de busca e analise dos trabalhos nas bases de dados.

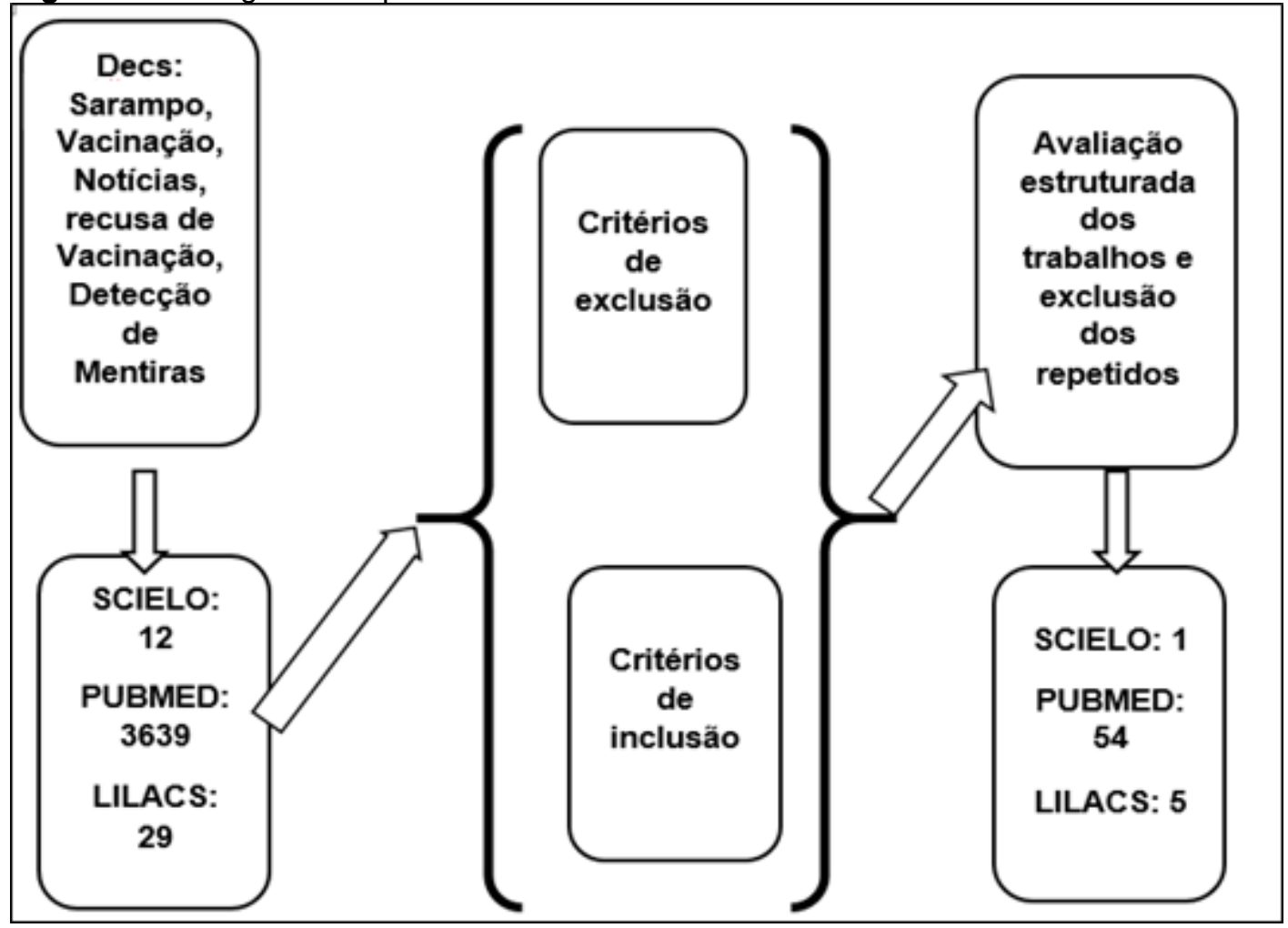

Fonte: Almeida HS, et al., 2021.

\section{DISCUSSÃO}

O sarampo surge como pauta importante no período de 2013, devido aos surtos concomitantes em várias partes do mundo (DA SILVA FV e DA SILVA JÚNIOR J, 2019; MELLO JN, et al., 2014). A abordagem clínicoepidemiológica da doença é observada nos trabalhos de Pereira JÁ, et al. (2013), Mello JN, et al. (2014), Borba RCN, et al. (2015), Ishikawa EKS e Gomide LMM (2015). No Brasil, o surto de sarampo atual seria resultado, principalmente, do aumento de indivíduos suscetíveis à doença, seja pelo esquema vacinal incompleto ou pelo comportamento de risco da não vacinação (BRANCO VGC e MORGADO FEF, 2019).

Em um mapeamento epidemiológico, realizado em 2013, referente ao ano anterior (2012), observou-se 8.230 casos de sarampo na Europa, com maior prevalência em cinco países: França, Itália, Romênia, Espanha e Reino Unido (EUROPEAN CENTER FOR DISEASE PREVENTION AND CONTROL, 2013).

As causas para as quedas em taxas de coberturas vacinais são exploradas em diversos trabalhos selecionados neste estudo. A avaliação de programas de coberturas vacinais em localidades especificas é observada no estudo de Gianfredi V, et al. (2019) que avaliou as taxas de vacinação na Itália, pelo período de 2015-2017. Priyadharshini AJ (2019) também apresenta trabalho semelhante, referente à cidade de Tamil Nadu, na Índia, em fevereiro de 2017, onde apontam os principais motivos de recusa da vacina, que foram os falsos rumores de efeitos adversos $(53,3 \%)$, e a falta de informações sobre os mesmos efeitos adversos $(47,5 \%)$.

Callender D (2016) apresenta uma breve construção histórica sobre o movimento de hesitação vacinal, apontando como marco recente um debate realizado no dia 16 de setembro de 2015, em Simi Valley, 
Califórnia, Estados Unidos, onde o então candidato à presidência, Donald Trump, manteve dialogo com dois representantes da saúde, a respeito das vacinas: benefícios e malefícios, onde as ideias associadas aos eventos adversos por imunização foram mais aceitas por parte significativa do público do que as defendidas pelos profissionais de saúde (CALLENDER, D., 2016).

Em revisão de literatura realizada por Phadke VK, et al. (2016), nos EUA, referente aos movimentos de hesitação e recusa vacinal, objetivando associar os achados aos surtos de sarampo e coqueluche no ano de 2016, observou-se em 18 estudos publicados sobre sarampo um total de 1416 casos da doença, sendo que $56,8 \%$ não tinham histórico de vacinação, dos quais $70,6 \%$ deles tinham comportamento de hesitação, por razões religiosas, filosóficas ou ideológicas.

Um estudo realizado por Martinez L, et al. (2016), avaliou uma ferramenta de análise de profissionais médicos da atenção primária, em relação à vacina, desenvolvida pela Société Française de Médecine Générale (SFMG), para validação da mesma. O questionário Determinantes das Intenções a Vacinar (DIVA (C), tornou-se uma ferramenta validada, possibilitando a sua aplicação em profissionais da área e a expansão para a população como um todo.

Greenberg J, et al. (2017) em revisão de um estudo desenvolvido pelo Departamento de Saúde Pública de Toronto, Canadá, em 2017, avaliaram a aplicação de um questionário on-line, aplicado para 1000 pais canadenses com crianças de 5 anos ou menos, evidenciando ao final da pesquisa que a maioria (92\%) considerava as vacinas seguras e eficazes e acreditava nos relatos médicos e de autoridades de saúde pública. No entanto, $28 \%$ deles acreditavam ou não tinham certeza se existiria um vínculo entre vacinas e autismo, temendo que as vacinas possam prejudicar seus filhos (27\%) ou acreditando que a indústria farmacêutica estaria por trás do esforço por imunização (33\%).

Um outro estudo, de Mrozek-Budzyn D, et al. (2017), partilha da mesma temática, porém, tem como objetivo determinar o conhecimento de 177 mães puérperas, em dois hospitais de Cracóvia e Myslenice, nos anos de 2014 e 2015, sobre doenças preveníveis por vacina, por meio de questionário estruturado.

O estudo de Arif $\mathrm{N}$, et al. (2018), avaliando como informações sobre vacinação, de caráter negativo, influenciam decisões acerca do assunto, verificou o número de informações sobre vacinas, disponíveis online, 20 anos após a publicação de artigo pelo The Lancet, em 1998, associando vacinas e autismo como consequência das mesmas, e utilizou o indicador padrão de qualidade das informações em saúde, o escore JAMA, para avaliar seu índice básico de confiabilidade. Entre $12 \%$ e $24 \%$ dos sites tinham uma posição negativa em relação às vacinas, enquanto a maioria dos sites era pró-vacina (43\%-70\%).

Por envolver o usuário de informações disponíveis em mídias sociais, a caracterização de seus perfis ajuda a estabelecer medidas de abordagem, baseadas nos aspectos de acesso e compartilhamento de informações (DA SILVA FV e DA SILVA JÚNIOR J, 2019; GONZAGA JD e DOS SANTOS AOC, 2019; MANSO MEG, et al., 2019). Yom-Tov E e Fernandes-Luque $L$ (2014), apresentaram a construção desse perfil do usuário baseado no acesso a sites específicos vinculados à temáticas diferentes (movimento antivacinação, domínios midiáticos do governo, informações farmacêuticas e médicas), concluindo que tanto indivíduos previamente contra vacinação, quanto a favor, acessavam os mesmos sites. No mesmo sentido, estão outros artigos, a exemplo de Ferro A, et al. (2015).

Radzikowski J, et al. (2016), analisaram 669.136 tweets sobre vacinação, nos EUA, entre fevereiro a março de 2015, avaliando termos em destaque, a taxa de replicação desses mesmos termos e as associações sociodemográficas desses usuários. O sarampo foi o termo mais encontrado nos tweets sobre vacinação, dados compatíveis com o surto de sarampo existente nos EUA no início de 2015 (RADZIKOWSKI J, et al., 2016).

As características dos boatos e fake news envolvendo a saúde são bastante únicas e devem ter políticas públicas de enfrentamento mais específicas (GONZAGA JD e DOS SANTOS AOC, 2019). Algumas das soluções observadas nesta presente pesquisa apresentavam caráter coercitivo, como o estudo de D'Ancona F et al. (2019), que avalia os efeitos, dois anos depois, da aplicação de uma lei compulsória de vacinação em julho de 2017, na Itália, em decorrência do surto anterior de sarampo. 
A maioria das soluções apresentadas nos estudos estava relacionada à melhora de técnicas de educação em saúde, principalmente as que possibilitam o uso de tecnologia de informação, como pode ser visto nos trabalhos de Horne Z, et al. (2015), Ferro A, et al. (2015) e Fadda M, et al. (2018).

A qualidade da informação em saúde na web é um aspecto importante, com a necessidade de avaliação mais profunda, já que há um déficit significativo sobre monitoramento e controle sobre o que é publicado e existe uma variabilidade enorme dentro do espectro de conhecimento prévio do usuário para emitir juízo de valor adequado sobre o conteúdo (GONZAGA JD e DOS SANTOS AOC, 2019). No Brasil, os órgãos de saúde como o Ministério da Saúde e o Conselho Federal de Medicina (CFM), trabalham no sentido de regulamentar as informações divulgadas na Web, sustentando a necessidade de mudança no papel de revisão das informações em saúde pelos pares, através de modelos de verificação da qualidade da informação como códigos de ética e selos de certificação de qualidade (LOPES IL, 2004).

\section{CONSIDERAÇÕES FINAIS}

A produção acadêmica mundial no período de 2013 a 2019, referente às temáticas de sarampo, imunização contra o mesmo (vacinação) e a influência de movimentos como hesitação, recusa ou antivacinação, fake news e pós-verdade, foi significativa, tendo sido encontrados 60 artigos. Dentre as publicações sobre o tema, o Brasil possui $5 \%$ do total. Os trabalhos de pesquisa aplicados (exploratória, quantitativa-descritiva e experimental) representaram a maior percentagem (75\%). As causas para as quedas em taxas de coberturas vacinais são exploradas em diversos trabalhos selecionados neste estudo, apontando que deve haver abordagem adequada junto à população, a fim de desmistificar as fake news.

\section{REFERÊNCIAS}

1. ARIF N, et al. Fake news or weak science? Visibility and characterization of anti-vaccine webpages returned by Google in different languages and countries.Frontiers in immunology, 2018; 9: 1215, 2018.

2. BORBA RCN, et al. The re-emergency and persistence of vaccine preventable diseases. Anais da Academia Brasileira de Ciências, 2015; 87(2): 1311-1322

3. BRANCO VGC, MORGADO FEF. O Surto de Sarampo e a Situação Vacinal no Brasil. Revista de Medicina de Família e Saúde Mental, 2019; 1 (1): 74-88.

4. BRASIL. MINISTÉRIO DA SAÚDE (BR). Situação do Sarampo no Brasil - 2018a. Informe No 14| $2017 / 2018$. Disponível em: http://portalarquivos2.saude.gov.br/images/pdf/2018/julho/19//nforme-Sarampo142.pdf. Acesso em: 22 out 2019.

5. BRASIL. Ministério da Saúde. Sarampo: Situação Epidemiológica; 2018b; Brasília. Cobertura vacinal. Brasília: Ministério da Saúde; 2018.

6. BRASIL. Ministério da Saúde. Secretaria de Vigilância em Saúde. Coordenação-Geral de Desenvolvimento da Epidemiologia em Serviços. Guia de Vigilância em Saúde. Ministério da Saúde, Secretaria de Vigilância em Saúde, Coordenação Geral de Desenvolvimento da Epidemiologia em Serviços. 3. ed. Atual. Brasília: Ministério da Saúde, 2019.

7. CALLENDER D. Vaccine hesitancy: more than a movement. Human vaccines \& immunotherapeutics, 2016; 12(9): 2464-2468.

8. D'ANCONA $\mathrm{F}$, et al. The law on compulsory vaccination in Italy: an update 2 years after the introduction. Eurosurveillance,2019; 24(26): 1900371.

9. DA SILVA FV, DA SILVA JÚNIOR J. Mentiras sinceras (não) me interessam: estratégias biopolíticas do Ministério da Saúde no combate às fake news. Revista Intersecções, 2019; 12 (27): 226-246.

10. EUROPEAN CENTER FOR DISEASE PREVENTION AND CONTROL, STOCKHOLM, 2013 - Surveillance report: Measles and rubella monitoring, February 2013. Disponível em: www.ecdc.europa.eu/en/activities/surveillance/euvac/ data/pages/reports.aspx. Acesso em 05 nov. 2019.

11. FADDA M, et al. Addressing issues of vaccination literacy and psychological empowerment in the measles-mumpsrubella (MMR) vaccination decision-making: a qualitative study. BMC Public Health, 2015; 15(1):836.

12. FERRO A, et al. Monitoring the web to support vaccine coverage: results of two years of the portal VaccinarSì. Epidemiol Prev, 2015; 39(4 Suppl 1): 88-93.

13. GENESINI S. A pós-verdade é uma notícia falsa. Revista USP, 2018; 116: 45-58.

14. GIANFREDI V, et al. Polio and measles: reasons of missed vaccination in Italy. Ann Ig, 2019; 31: $191-201$.

15. GONZAGA JD, DOS SANTOS AOC. Fake news, desinformação e saúde pública: estudo sobre a abordagem do tema 'vacina' na agência de checagem Lupa. XXIV Congresso de Ciências da Comunicação na Região Sudeste. 2019: 115.

16. GREENBERG J, et al. Vaccine hesitancy: in search of the risk communication comfort zone. PLoS currents, $2017 ; 9$. 
17. HENRIQUES CMP. A dupla epidemia: febre amarela e desinformação. RECIIS - Revista Eletrônica de Comunicação, Informação e Inovação em Saúde, Rio de Janeiro, 2018; 12(1): 9-13.

18. HORNE Z, et al. Countering antivaccination attitudes. Proceedings of the National Academy of Sciences, 2015; 112(33): 10321-10324.

19. ISHIKAWA EKS, GOMIDE LMM. Doenças emergentes e reemergentes: um problema do passado que persiste no presente. Revista Intersaúde, 2019; 1(1): 59-72.

20. LAGO EG. Hesitação/recusa vacinal: um assunto em pauta - Editorial (Vaccine hesitancy/refusal: a current issue Editorial). Rev Sci Med, 2018; 28(4): 1-3.

21. LOPES IL. Novos paradigmas para avaliação da qualidade da informação em saúde recuperada na Web. Ciência da Informação, 2004; 33(1): 81-90.

22. MANSO MEG, et al. Fake News e Saúde da Pessoa Idosa. Revista Longeviver, 2019; 2: $19-25$.

23. MARTINEZ L, et al. Scoring and psychometric validation of the 'Determinants of Intentions to Vaccinate'(DIVAC) questionnaire. BMC family practice, 2016; 17(1): 143.

24. MELLO JN, et al. Panorama atual do sarampo no mundo: Risco de surtos nos grandes eventos no Brasil. J. bras. med, 2014; 102(1):33-40.

25. MOTHER D, et al. The PRISMA Group (2009). Preferred Reporting Items for Systematic Reviews and Meta-Analyses: The PRISMA Statement. PLoS Med 6(7): e1000097.

26. MROŻEK-BUDZYN D, et al. What mothers know about vaccine preventable diseases?. Przeglad epidemiologiczny, 2017, 71(4): 595-602.

27. NAZARETH RT. SAÚDE E MÍDIA SOCIAL: As fake News que matam. Unisanta Law and Social Science, 2019; 7(3): 593-604.

28. PEREIRA JÁ, et al. Contagious comments: what was the online buzz about the 2011 Quebec measles outbreak?. PLoS One, 2013, 8(5): e64072.

29. PHADKE VK, et al. Association between vaccine refusal and vaccine-preventable diseases in the United States: a review of measles and pertussis. Jama, 2016, 315(11): 1149-1158.

30. PRIYADHARSHINI AJ. Coverage survey of Measles-Rubella mass vaccination campaign in a rural area in Tamil Nadu. Journal of family medicine and primary care, 2019, 8(6): 1884.

31. RADZIKOWSKI J, et al. The measles vaccination narrative in Twitter: a quantitative analysis. JMIR public health and surveillance, 2016, 2(1): e1.

32. RIBEIRO C, et al. Achados Epidemiológicos Recentes e Implicações para a Prática Clínica. Almanaque Multidisciplinar de Pesquisa, 2015, 1(2): 1-13.

33. SAITA HS. Os movimentos antivacinais e a recusa das vacinas: uma revisão integrativa. Repositório São Lucas, 2019.

34. SATO APS. Qual a importância da hesitação vacinal na queda das coberturas vacinais no Brasil? Rev. Saúde Pública, 2018, 52: 1-9.

35. SILVA LM, et al. Impacto da pós-verdade em fontes de informação para a saúde. Revista Brasileira de Biblioteconomia e Documentação, 2017, 13: 271-287.

36. SILVA SS. Sarampo na era da eliminação no Brasil: estudo de surtos recentes baseado no sequenciamento da região não codificante do genoma do vírus. Dissertação [Mestrado]. Medicina Tropical, Instituto Oswaldo Cruz, Fundação Oswaldo Cruz, Rio de Janeiro, 2018; $160 \mathrm{f}$.

37. SBIM-SOCIEDADE BRASILEIRA DE IMUNIZAÇÕES. Sarampo: Diagnóstico, notificação e prevenção. NOTA TÉCNICA 16/07/2018. Disponível em https://sbim.org.br/images/files/nota-tecnica-conjunta-saramposbimsbisbp20180716.pdf Acesso em 05 out 2020.

38. WAKEFIELD AJ, et al. Ileal-lymphoid-nodular hyperplasia, non-specific colitis, and pervasive developmental disorder in children. The Lancet; 1998; 351 (9103): 637-641.

39. XAVIER AR, et al. Diagnóstico clínico, laboratorial e profilático do sarampo no Brasil. Jornal Brasileiro de Patologia e Medicina Laboratorial, 2019, 55(4): 390-401.

40. YOM-TOV E, FERNANDEZ-LUQUE L. Information is in the eye of the beholder: Seeking information on the MMR vaccine through an Internet search engine. In: AMIA Annual Symposium Proceedings. American Medical Informatics Association, 2014,:1238. 